\title{
Restitution de récit et nouvelle littératie scolaire. Étude comparée de productions orales d'élèves issus d'établissements scolaires de milieux contrastés
}

\author{
Catherine Delarue-Breton ${ }^{1}$ et Élisabeth Bautier ${ }^{2}$ \\ ${ }^{1}$ Université Paris-Est, EA 4384 Circeft, 94010 Créteil Cedex, France \\ ${ }^{2}$ Université Paris 8 Vincennes-Saint-Denis, EA 4384 Circeft, 93200 Saint-Denis, France
}

\begin{abstract}
Résumé. Différents travaux font senétat d'un lien entre la manière dont les élèves s'approprient les enjeux des activités scolaires, notamment la manière dont ils interprètent les énoncés et textes qui leur sont proposés en classe, et leur mode de socialisation familiale.

Nous avons cherché à vérifier l'existence d'une corrélation entre le milieu social et les dispositions des élèves en lien avec les pratiques scolaires littératiées, à travers une activité pratiquée à tous les niveaux de la scolarité du premier degré, la lecture d'albums de jeunesse.

La comparaison des restitutions de récit effectuées par les élèves (6-7 ans) de deux établissements scolaires fréquentés par des élèves de milieux très contrastés fait en effet apparaitre une différence importante dans la nature des productions de ces deux populations, qui concerne autant les choix d'évènements ou d'éléments à restituer, que la manière de reconstituer un récit dans une forme qui relève de la littératie scolaire, sans doute en lien avec une interprétation différenciée des réquisits de l'activité. Cette communication participe donc de travaux sur la complexification de la littératie scolaire et des inégalités sociales qu'elle est susceptible d'entraîner au sein même des pratiques scolaires.
\end{abstract}

\section{Contexte}

Les travaux de notre équipe (CIRCEFT-Escol) font état depuis plus d'une vingtaine d'années du caractère socialement situé des inégalités scolaires [1]. Les chercheurs du réseau ReSEIDA ${ }^{1}$ se sont penchés plus précisément sur le rôle des pratiques enseignantes et des activités proposées aux élèves dans l'aggravation ou la réduction des inégalités d'apprentissage [2].

Nous avons pour notre part cherché à comprendre le rôle éventuel joué par les supports proposés aux élèves dans l'aggravation des difficultés lors des activités en lien avec la littératie scolaire, notion sur laquelle nous reviendrons plus loin.

Il s'agit ici de :

- Montrer le caractère socialement situé des inégalités scolaires à travers des activités comparées de lecture au cycle 2 (élèves de 6-7 ans).

- Évaluer le rôle du caractère composite des supports dans les difficultés rencontrées par les élèves.

\footnotetext{
${ }^{1}$ RESEIDA : REcherches sur la Socialisation, l'Enseignement, les Inégalités et les Différenciations dans les Apprentissages. Ce réseau a été créé en 2001 à l'initiative d'Élisabeth Bautier et Jean-Yves Rochex (CIRCEFT-Escol) et fait intervenir des chercheurs de disciplines différentes sur la manière dont se coconstruisent les inégalités au sein même de la classe.
}

This is an Open Access article distributed under the terms of the Creative Commons Attribution License 4.0, which permits unrestricted use, distribution, and reproduction in any medium, provided the original work is properly cited. 
Concernant ce dernier point, nous souhaitons attirer l'attention du lecteur sur une dimension importante de notre propos. Évaluer le rôle du caractère composite des supports dans les difficultés potentielles des élèves ne signifie en aucun cas déplorer l'usage de ces supports en classe : notre société se complexifie, les modes de littératie qu'elle convoque se complexifient également, il nous apparaît donc évident que des supports plus complexes doivent être exploités à l'école.

Notre recherche vise donc à comprendre un peu mieux les facteurs d'accroissement des inégalités scolaires, dont les enquêtes PISA nous rappellent régulièrement qu'elles ne cessent d'augmenter en France et d'identifier les éléments de cette littératie qui ne sont pas partagés par tous les élèves et, ce faisant, ce que désormais l'école doit enseigner.

\section{Aspects théoriques}

Trois notions clés constituent le soubassement théorique de cette recherche :

- La notion de coconstruction des inégalités scolaires [2-4], qui stipule que deux types de facteurs conjugués permettent de rendre compte des inégalités scolaires. D'une part, des facteurs liés au mode de socialisation familiale des élèves, certains modes de socialisation prédisposant plus favorablement que d'autres aux apprentissages littératiés scolaires ; d'autre part des facteurs liés à ce qui se joue au sein même de la classe, touchant les pratiques enseignantes et les dispositifs d'apprentissages proposés aux élèves, qui contribuent à la réduction ou au contraire à l'aggravation des inégalités liées au mode de socialisation familiale.

- La notion de support composite [5, 7-9], qui contribue à théoriser l'évolution des supports de travail proposés aux élèves lors des activités scolaires, qu'il s'agisse de fichiers, de manuels, d'ouvrages de littérature de jeunesse ou d'autres documents. Cette évolution se caractérise en effet par une plus grande hétérogénéité des documents, au plan sémiotique (textuel et iconique) comme au plan discursif (lecteur ciblé multiple, sources énonciatives plurielles), une délinéarisation du propos, au profit d'une organisation plus éclatée et fragmentée, discontinue, dont le fil conducteur demeure implicite.

- La notion de littératie scolaire, [9, 10] qui met l'accent sur des usages de l'écrit multimodaux, socialement et historiquement construits, ainsi que sur les transformations cognitives qu'ils nécessitent et qu'ils entraînent. Nous désignons ainsi par littératie scolaire [11] les exigences de lecture, de raisonnement et de connaissances qui supposent une familiarité avec la fréquentation des écrits scolaires actuellement proposés aux élèves et qui ont modifié l'apparence des manuels, des albums, des fiches de travail. Elle suppose l'utilisation par les élèves de documents complexes, visant l'élaboration d'un texte (oral ou écrit) de savoir. Ces outils de travail, présents dans les différentes disciplines et aux différents niveaux de la scolarité, mettent en jeu des registres cognitifs et culturels hétérogènes. Ils impliquent la mobilisation de processus cognitifs et de conceptions qui prennent la langue et les objets du monde comme objets d'analyse, de questionnement, de travail (et non d'expériences ou d'usages spontané). Notamment, le document est à penser ou à concevoir comme document à interpréter, engageant la construction de significations (et non « seulement » comme un texte à comprendre) grâce à des mises en relation d'objets au sein du texte et au delà de celui-ci, afin de construire des réseaux de significations. Il s'agit donc de prendre en compte le texte dans sa globalité, ce qui passe par la mise en relation d'éléments linguistiques et discursifs qui peuvent être éloignés les uns des autres. Mais il s'agit également de traiter des signes graphiques, en prenant en considération la logique plurisémiotisée qui sous-tend leur organisation spatiale et scripturale (espace, caractères gras ou non, soulignements, tableaux, schémas, textes...), ainsi que la matérialité de la langue écrite. Cette plurisémiotisation implique donc la connaissance d'un système de représentation du monde et de raisonnements en lien avec l'écrit, connaissance dont on sait aujourd'hui qu'elle est inégalement partagée. 


\section{Aspects méthodologiques}

Les données analysées sont constituées à la fois d'ouvrages de littérature de jeunesse (albums), appartenant à la catégorie des supports composites, et d'entretiens avec des élèves après lecture magistrale ou autonome (en fonction du niveau des élèves en lecture) de ces mêmes supports. Un autre volet de la recherche prend en charge l'étude du rôle des pratiques enseignantes dans l'appropriation de ces supports par les élèves ; notre but pour ce volet-ci a été de chercher à mettre en évidence l'hétérogénéité importante des difficultés a priori des élèves confrontés à ce type de support, en lien avec leur milieu social.

Nous présenterons ici l'analyse de trois albums, d'autres sont en cours d'analyse.

Les entretiens avec les élèves ont été effectués dans deux types d'établissements scolaires socialement contrastés d'Île-de-France : d'une part, une école située en $\mathrm{ZEP}^{2}$, d'autre part, une école recrutant des élèves au capital social et culturel élevé, très homogène. Plus de cent cinquante entretiens sont en cours d'analyse, la moitié environ a été analysée plus finement pour cette contribution.

La méthodologie retenue pour l'entretien est l'entretien semi-directif, au cours duquel les mêmes questions ont été posées aux élèves des deux types d'établissement, l'une d'entre elle invitant à la restitution du récit lu (sur le modèle « raconte-moi l'histoire de... »), les autres concernant divers aspects du récit en lien avec le carcatère composite de l'ouvrage. L'analyse des restitutions est en cours, les réponses aux autres questions ont été comparées terme à terme.

Sans doute, une analyse comparée des pratiques pédagogiques mises en œuvre dans les deux types de classe permettrait éventuellement de rendre compte pour partie du rôle de la socialisation scolaire dans les différences constatées. Nos travaux se sont d'ailleurs attachés à montrer à plusieurs reprises le rôle particulièrement différenciateur des pratiques enseignantes dans la réduction ou l'aggravation des inégalités scolaires (voir par exemple Rochex \& Crinon [2], ou Delarue-Breton [12]).

Cependant, dans la mesure où ces élèves de CE1, qui sont scolarisés depuis plus de 4 ans pour la plupart d'entre eux, ont été pris en charge par des enseignants différents et ont donc bénéficié de modes de socialisation scolaire hétérogènes depuis le début de leur scolarité - et donc d'histoires scolaires différentes - notre objectif ici a été de chercher à identifier les points de basculement récurrents. Nous avons ainsi centré notre approche sur les lieux précis qui sont susceptibles de faire régulièrement obstacle à une production de signification pertinente (autrement dit qui ne contredise pas les droits du texte et qui rende compte de sa visée pragmatique) de la part d'élèves de milieux défavorisés, confrontés à ce type de supports, indépendemment du mode de socialisation et d'apprentissage scolaires. Un autre volet de cette même recherche vise à comprendre les effets de la socialisation scolaire, et les premiers résultats semblent indiquer que la spécificité de ces nouveaux types de support est peu prise en compte par les enseignants.

Nous avons donc procédé à une analyse croisée des albums, afin de déterminer le degré ou l'importance de leur caractère composite, et des productions orales des élèves lors des entretiens, et plus particulièrement en ce qui concerne la prise en charge, dans leurs propos, du caractère composite de ces documents.

\section{Qu'est-ce qu'un document composite ? Analyse comparée de trois albums de littérature de jeunesse}

Les documents composites sont des documents hétérogènes et fragmentés, constitués d'atomes d'information ou «nœuds », reliés plus ou moins explicitement par des liens en un réseau où l'utilisateur navigue selon des parcours qu'il détermine lui-même parmi un grand nombre de possibles [5].

\footnotetext{
2 ZEP : Zone d'Éducation Prioritaire. Les ZEP sont constituées d'un ensemble d'établissements scolaires relevant d'un projet commun visant à lutter contre les inégalités liées à l'appartenance sociale défavorisée, voire très défavorisée des élèves.
} 


\section{SHS Web of Conferences}

Leurs traits caractéristiques conjoints sont les suivants :

- Hétérogénéité sémiotique (pluralité des systèmes, et à l'intérieur d'un système, pluralité des codes).

- Hétérogénéité discursive (enchevêtrement de voix, situations de double énonciation, lecteur supposé multiple : naïf, cultivé, voire adulte).

- Discontinuité (absence de linéarité susceptible de guider la lecture et relations entre des éléments disjoints et hétérogènes à construire par le lecteur).

Nous ne retenons pas, en l'état actuel de la recherche, comme document composite des documents qui présenteraient l'un ou l'autre de ces traits ; la qualification composite renvoie donc aux trois traits présents ensemble sur le document.

\subsection{Album 1 : Philippe Matter (1996), Mini-Loup à l'école. Paris : École des loisirs}

Ce premier ouvrage est celui que nous rangerons dans la catégorie des ouvrages peu composites, au regard des deux autres ${ }^{3}$.

Il présente en effet une hétérogénéité sémiotique faible, avec un rapport texte image redondant à une exception près, et une composition de l'ouvrage récurrente, de type double page avec texte dialogué + illustration.

Du point de vue discursif, on observe également une hétérogénéité faible : le lecteur pressenti est un lecteur enfant naïf [7], susceptible de s'identifier aisément au personnage principal, Mini-Loup. L'alternance discours/récit est clairement marquée, et les voix sont aisément identifiables (locuteur personnage soutenu par l'image, alternant avec un narrateur externe).

L'ensemble présente donc une discontinuité faible également, et la narration est de type saturé [13]. ${ }^{4}$

\subsection{Album 2 : Christian Jolibois et Christian Heinrich (2005), Charivari chez les p'tites poules. Paris : Pocket jeunesse}

À l'opposé de l'exemple précédent, cet ouvrage apparaît comme présentant un caractère composite affirmé, au plan sémiotique comme au plan discursif, et une discontinuité marquée.

Au plan sémiotique, l'hétérogénéité du document concerné est importante : on observe ainsi, du point de vue iconique, un rapport texte/image pluriel (parfois redondant, parfois complémentaire, parfois contradictoire), une multiplicité des formats et des cadrages, une densité forte d'éléments présents sur les images. Du point de vue du texte, les typographies employées sont variées, et les propos tenus se situent à des endroits divers et non nécessairement récurrents de la page.

Au plan discursif, l'hétérogénéité du document est également particulièrement remarquable : on note des formes de discours indirect libre au delà de l'alternance récit/discours, et les voix, qui semblent s'organiser en deux camps, présentent en réalité à l'intérieur de chaque camp des nuances qui rendent leur appartenance parfois peu identifiable ; enfin, le lecteur pressenti est mixte, tantôt enfant naïf, tantôt enfant cultivé, tantôt adulte.

Cet ouvrage présente donc une discontinuité forte, impliquant des cheminements possibles plurivoques.

\footnotetext{
${ }^{3}$ Nous précisons en effet que cette hiérarchisation est relative, et ne saurait s'entendre dans l'absolu : les ouvrages sont considérés comme plus ou moins composites les uns par rapport aux autres.

${ }^{4}$ Poslaniec (ibid.) définit par ce terme un type de narration suscitant peu une activité interprétative du lecteur, relevant d'une postulation fermée, c'est-à-dire pourvue d'un narrateur omniscient, avec un maximum d'éléments explicites (peu de blancs dans la narration), et des personnages emblématiques et aisément classables plutôt qu'ambivalents.
} 


\subsection{Album 3 : Geoffroy de Pennart (1999), Le loup sentimental. Paris : Kaléidoscope.}

Comparé aux deux précédents, cet ouvrage nous apparaît comme pouvant se ranger dans une catégorie intermédiaire. Il présente en effet une hétérogénéité sémiotique moyenne, où le rapport texte/image est souvent redondant, mais où l'illustration renvoie parfois à plusieurs situations en même temps (hétérogénéité temporelle); la composition de l'album est du même type que pour l'album 1. En termes d'hétérogénéité discursive, si l'alternance récit/discours est clairement marquée, le texte présente une forme de dialogisme (au sein de laquelle les situations se font écho) qui n'est donc pas immédiatement saisissable, et le lecteur pressenti est mixte (simultanément lecteur naïf et lecteur enfant cultivé, puisque, par exemple, l'allusion aux différents contes de fées est récurrente tout au long de l'ouvrage).

\section{Que disent les productions orales des élèves confrontés à ces supports ? L'exemple du Loup sentimental}

Nous ne pouvons évoquer ici les productions orales des élèves concernant chacun des trois albums; nous avons donc choisi pour cette contribution d'exploiter les entretiens qui ont fait suite à la lecture par les élèves de l'album intitulé Le loup sentimental. Nous en donnons plus loin le détail. En ce qui concerne l'étude des productions orales des élèves des milieux ZEP et favorisé à la suite de la lecture du premier ouvrage (Mini-Loup à l'école), nous avons constaté une réussite générale des élèves sur l'ensemble des questions, sans différence particulièrement notable entre les deux populations concernant la restitution du récit et l'exactitude des évènements mentionnés. Inversement, les productions comparées des élèves après lecture du deuxième ouvrage (Charivari chez les p'tites poules) montrent un écart tellement important entre les deux populations qu'il est parfois malaisé d'identifier les points de basculement: la recherche en cours semble montrer une prise en charge de l'évènementiel par les élèves de ZEP, au détriment des effets d'échos et d'autres aspects plus littéraires de l'ouvrage. Les productions orales qui ont suivi la lecture du Loup sentimental ont quant à elles permis d'identifier pour partie en quoi, précisément, le caractère composite d'un document est susceptible de contribuer à l'aggravation des inégalités si l'école n'y prend garde, et de faire apparaître des éléments précis du support à partir desquels les deux populations diffèrent.

\subsection{Le protocole}

Voici le résumé de l'histoire du Loup sentimental :

Un loup nommé Lucas devenu grand quitte sa famille et part pour la forêt ; les rencontres successives qu'il y fera lui rappelleront la séparation d'avec les siens, ce qui l'empêchera de dévorer ses proies habituelles (trois petits cochons, sept biquets, Chaperon rouge, Petit Pierre etc.), qui figurent pourtant sur la liste de ce qu'il peut manger, qui lui a été remise par son père ${ }^{5}$.

Dix questions ont donc été posées à chacun des élèves, que nous reproduisons ci-dessous. Trois d'entre elles (en gras) retiendront ici notre attention :

1. Quel est ton prénom?

2. Connaissais-tu le livre avant que je ne le lise tout à l'heure?

3. Raconte-moi l'histoire du Loup sentimental.

4. Page 10 : De quelle liste s'agit-il ? Comment l'a-t-il obtenue ?

5. Page 19 : Pourquoi pense-t-il à ses frères à ce moment-là de l'histoire ?

\footnotetext{
5 Voici ce qui figure sur la liste, intitulée Bon à manger : chèvre et chevreaux, Chaperon rouge, petits cochons, Pierre, Petit Poucet et ses frères. Le loup, qui ne mangera aucune de ces proies et barrera à chaque rencontre le nom de celui qu'il ne mange pas, ajoutera lui-même sur cette liste le mot ogre, après avoir dévoré ce dernier.
} 


\section{Page 23 : Pourquoi dit-il à Pierre qu'on ne désobéit jamais à son grand-père ?}

7. Page 33 : Pourquoi certains mots de la liste sont-ils barrés ?

8. Page 33 : Pourquoi le loup n'a-t-il pas mangé ces personnages alors qu'ils sont sur sa liste ?

9. Comment expliques-tu le titre ? Que veut-il dire?

10. Sais-tu ce que signifie le mot sentimental ? Peux-tu me le dire?

Les questions qui font l'objet de l'analyse (question 4, 5 et 6) concernent toutes les trois l'effet d'écho entre la situation initiale, où le loup quitte les siens, et les diverses rencontres qu'il fera dans la forêt. Nous avons donc cherché à comprendre dans quelle mesure cet écho avait pu non seulement être perçu par les élèves, mais aussi restitué spontanément, et donc identifié - ou non - par ces derniers comme phénomène inhérent à la lecture d'un texte. En effet, il s'agit alors dans l'activité de lecture de mémorisation et de mise en relation d'éléments qui manifestent que l'élève prend le texte dans sa globalité pour produire des significations, et en construire une interprétation.

\subsection{Réussites communes aux deux populations d'élèves}

Ces réussites concernent les questions 4 (De quelle liste s'agit-il ? Comment l'a-t-il obtenue ?) et 5 (Pourquoi pense-t-il à ses frères à ce moment-là de l'histoire ?).

En ce qui concerne la question 4 , la liste dont il est question est celle que le père du loup a remise à notre héros, Lucas, au moment du départ de celui-ci pour la forêt. Voici le propos tenu par le père de Lucas à son fils : «Tiens, voici la liste de tout ce que tu peux manger».

La quasi-totalité des élèves reconnaissent la liste et savent indiquer aussi bien sa provenance que sa fonction, en milieu favorisé comme en ZEP. Il est à noter cependant que nombre d'élèves de milieux très favorisés mentionnent spontanément cette liste et son origine lors de la restitution du récit (question 1 du protocole), ce qui n'est que rarement le cas en ZEP.

Du point de vue du support, on observe que la liste constitue en quelque sorte l'un des fils conducteurs du propos, puisqu'elle apparaît dans la main de Lucas sur chaque double page de l'album (à une exception près), et qu'elle est également mentionnée tout au long de l'ouvrage, dans les propos du loup, à travers des formules du type « tu es sur ma liste, je vais donc te manger».

Pour ce qui est de la question 5, celle-ci renvoie à l'émotion éprouvée par le loup lorsqu'il rencontre les trois petits cochons. Alors qu'il leur indique qu'il va les manger, ceux-ci se mettent à chanter, pour se dire adieu, la même chanson que ses propres frères (qui sont trois également) lui ont chantée au moment de son départ pour la forêt. Le lien entre les deux moments du récit est donc simultanément perceptible au plan visuel et plan textuel. Les deux images sont en effet très comparables ( 3 cochons / 3 frères de Lucas jouant de la musique et chantant la même chanson), et le texte de la chanson est présenté dans les deux cas au sein d'un phylactère raccordé à deux des personnages, le troisième jouant d'un instrument à bouche, et ne pouvant donc chanter. Voici les deux pages de l'album, placées côte à côte.

\subsection{Différences entre les deux populations d'élèves}

Ces différences se manifestent dans les productions orales des élèves notamment par la mention ou l'absence de mention du lien entre la scène initiale, où Lucas se sépare de son grand-père, qui lui fait alors présent de sa montre et l'invite à ne pas la refuser au motif qu' « on ne désobéit jamais à son grand-père », et la scène écho où Lucas rencontre Pierre, qui lui avoue fortuitement avoir désobéi à son grand-père pour venir le chasser, propos inconsidéré qui entraînera immédiatement le courroux du loup, qui rétorquera précisément au jeune Pierre «qu’on ne désobéit jamais à son grand-père ».

Les productions orales des élèves présentent ici des différences massives : tandis que $90 \%$ des élèves issus de milieu favorisé repèrent le lien qui unit les deux scènes, seulement $25 \%$ d'entre eux y parviennent dans l'établissement situé en ZEP. 


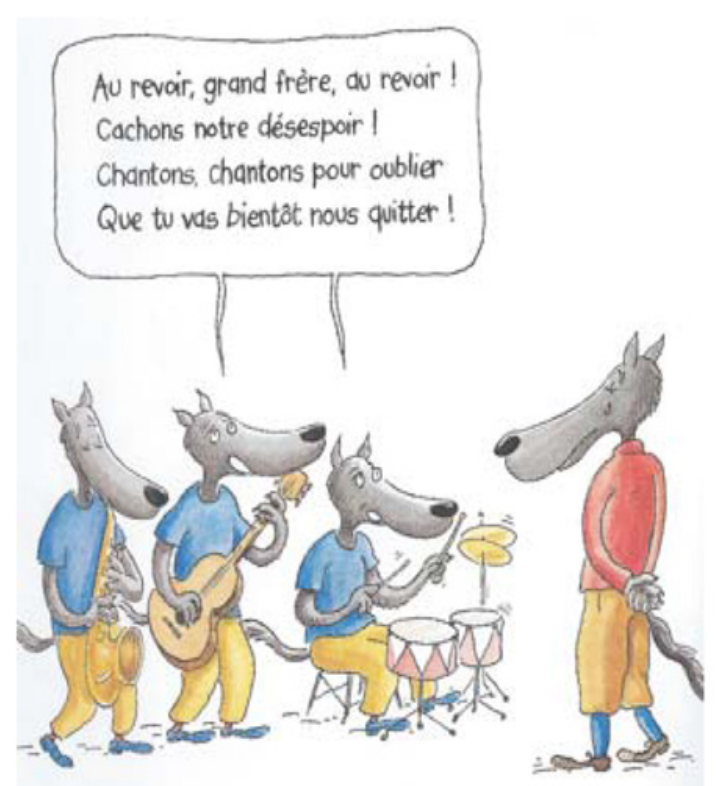

"Nous allons chanter pour fêter ton départ *, s'écrient ses jeunes frères qui s'exécutent aussitôt.

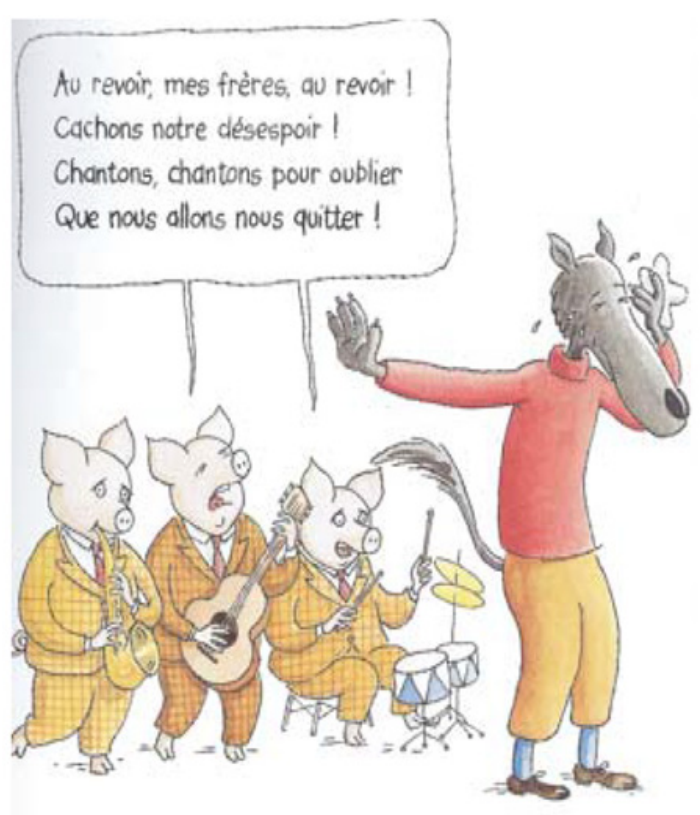

Lucas acquiesce. Mais, en les écoutant, il ne peut s'empêcher de songer à ses frères. "Filez avant que je ne me ravise ", grogne-t-il tout bouleversé.

Geoffroy de Pennart (1998). Le loup sentimental. Paris : Kaléidoscope (p. 6 et p.19).

Mais revenons sur l'analyse du support : le seul lien formellement attesté entre ces deux scènes réside dans la formule scandée comme un slogan «on ne désobéit jamais à son grand-père », qui apparaît lors de chacune d'elles. On peut donc considérer que ce lien est un lien explicite, attesté linguistiquement, même s'il n'est pas appuyé par d'autres éléments, au plan textuel ou iconique. Pourtant, ni le contexte du propos (entendu comme son environnement situationnel) ni son cotexte (entendu comme son environnement linguistique) n'invitent spécifiquement au rapprochement entre les deux scènes.

D'un côté, la scène initiale apparaît au plan contextuel comme un moment d'émotion contenue où la tendresse rivalise avec le chagrin, dans un moment à la fois redouté et attendu où l'aîné quitte le domicile familial : le père « soupire », la mère «s'attriste », la grand-mère l'invite à revenir souvent, et le grand-père exhorte son petit fils à accepter un présent d'une valeur symbolique forte : sa montre. Au plan cotextuel, le ton est également celui de la tendresse :

«Prends cette montre », bougonne son grand-père. "Je sais qu'elle t'a toujours fait envie ».

«Oh! non, Grand-père, c'est vraiment trop!»

"Pas d'histoire, on ne désobéit JAMAIS à son grand-père », rétorque le vieux loup. ${ }^{6}$

De l'autre, la scène « écho », où le loup rencontre Pierre, se présente sous un jour beaucoup plus brutal et moins contenu que la précédente : le cotexte sémantique du propos est violent, le loup « hurle de sa plus grosse voix », Pierre est « épouvanté »; au plan sémiotique, si les majuscules apparaissent déjà lors

${ }^{6}$ Geoffroy de Pennart (1999), Le loup sentimental, Paris, Kaléidoscope, p. 9. 
de la scène initiale (JAMAIS), c'est pour l'ensemble du propos du loup qu'elles sont employées lors de la seconde scène :

«ON NE DÉSOBÉIT JAMAIS À SON GRAND-PÈRE, TU M'ENTENDS ? » hurle Lucas de sa plus grosse voix. Pierre, épouvanté, prend ses jambes à son cou. ${ }^{7}$

Au plan iconique, la différence entre les deux moments est également importante : alors que la scène initiale montre une famille attendrie qui fait preuve d'une grande sollicitude à l'égard du voyageur (Lucas est dans les bras de sa grand-mère qui l'étreint, son grand-père lui sourit en lui offrant sa montre), la scène écho montre un loup hors de lui, la gueule grande ouverte et les sourcis froncés, désignant d'un doigt accusateur un Pierre épouvanté, les cheveux hérissés, lachant son fusil et accompagné dans sa fuite par ses acolytes (le canard et le petit oiseau) également horrifiés.

Enfin - et ce fait nous apparaît comme particulièrement révélateur d'une différence de positionnement des élèves concernant la prise en charge du texte en tant qu'objet d'analyse - nous observons que notre question (Pourquoi dit-il à Pierre qu'on ne désobéit jamais à son grand-père ?) a pu être entendue par les uns (hors ZEP) comme une question renvoyant au fonctionnement discursif global du texte, ce qui a abouti à des énoncés d'élève du type « parce que son grand-père lui a dit la même chose quand il lui a donné sa montre »; tandis que les autres l'ont entendue massivement comme une simple quête d'information locale (en ZEP), et y ont répondu en fournissant l'information qui se trouvait dans le cotexte immédiat du propos, dans des énoncés du type «parce que Pierre a désobéi à son grand-père », information effectivement fournie à la page précédente.

Il nous a ainsi paru particulièrement remarquable que, là où le texte reprend les mêmes mots, le cotexte et le contexte suffisent à brouiller le fil permettant de rapprocher les scènes, au point que seulement un quart des élèves de ZEP sont en mesure de le restituer. Discontinuité sémiotique et discontinuité discursive viennent ainsi perturber non seulement la linéarité du propos d'un bout à l'autre de l'ouvrage, mais aussi les effets d'écho entre les scènes qui présentent pourtant des éléments objectifs de continuité, à savoir les mots employés. En matière de production de significations, la contiguïté le cotexte - l'emporte donc sur la continuité - le contexte - pour le plus grand nombre d'élèves de l'établissement situé en ZEP, quand celle-ci n'est pas portée par une pluralité de marqueurs, d'ordres à la fois textuel et iconique.

\section{Conclusion}

Deux questions étaient au cœur de notre réflexion, la question des différences socialement situées d'une part, celle du rôle des documents composites dans les difficultés des élèves au cours des activités littératiées d'autre part.

Pour ce qui est de la première, nous pouvons affirmer une corrélation forte entre les modes d'approche de ces documents par les élèves et leur milieu social constitué ici par leur établissement d'origine dont l'homogénéité de la population est maximale : quand les uns prennent en compte leur dimension multimodale ou multidimensionnelle (effets d'écho, interaction texte image, références hypertextuelles etc.), d'autres s'inscrivent davantage dans une lecture évènementielle qui s'écarte peu de la successivité des événements, et prend appui sur des informations plus localisées et souvent plus iconiques que textuelles. Ces différences socialement situées renvoient donc notamment à l'hétérogénéité des modes de socialisation lectorale familiale ${ }^{8}$, les uns - ceux des familles à capital culturel et social élevé - acculturant très tôt les élèves à produire des significations à partir de supports

\footnotetext{
7 Ibid. p. 27.

${ }^{8}$ Voir également sur ce point les travaux de Bernstein [6], qui montrent qu'il peut exister un rapport à la littératie scolaire socialement construit, qui nécessite d'être pris en compte par l'école pour voir régresser les inégalités scolaires.
} 
hétérogènes. L'importance des écarts entre élèves (taux de réussite de $90 \%$ contre $25 \%$ pour les réponses à la question 6) invite ainsi non seulement à attirer l'attention sur le rôle de l'environnement dans la façon dont les élèves traitent cognitivement, linguistiquement, et culturellement des textes composites, mais aussi à faire état du caractère très précoce de l'action de l'environnement sur les apprentissages littératiés, ce qui conduit, pensons-nous, à devoir réinterroger les missions de l'école maternelle, et surtout les conditions dans lesquelles elle est susceptible de les remplir (ce qui ne signifie pas que l'école maternelle ne joue actuellement pas ce rôle : mais elle ne le joue sans doute pas aussi massivement qu'on pourrait le souhaiter).

Pour ce qui est de la seconde, qui concerne le rôle des supports composites dans les difficultés des élèves au cours des activités littératiées, la recherche présentée ici révèle aussi ce dont sont capables des élèves âgés d'environ six ans en matière de production de signification et d'élaboration de liens entre des éléments pas nécessairement continus, pas nécessairement de même nature, lorsque leur mode de socialisation familiale les y a entraînés. Ce qui signifie pour nous, ici encore, que c'est certainement bien avant l'entrée au CP que l'école peut contribuer à réduire les inégalités, y compris en ce qui concerne des activités en lien avec l'écrit et notamment avec les textes afin de construire chez les élèves les dispositions nécessaires pour travailler et construire des savoirs et des significations à partir des supports de travail proposés.

Enfin, cette étude révèle également que l'engouement - qui ne nous apparaît en aucun cas illégitime, nous le redisons avec force - pour ce type de support suppose cependant que leur spécificité soit prise en compte en classe, et donc que soient conçues et mises en œuvre des pratiques d'enseignement les concernant et concernant les difficultés spécifiques qu'ils présentent pour une partie de la population scolaire (par exemple élaboration collective de la linéarité du récit, prise en charge de sa recomposition autant que de sa décomposition, institutionnalisation des séances en lien avec la visée pragmatique du texte etc.). Les travaux de recherche en littérature ont déjà montré dans quelle mesure le lecteur peut être sollicité pour participer à la production de sens (nous pensons par exemple au texte considéré comme machine paresseuse d'Umberto Eco [14], qui suppose des lecteurs une coopération active dans l'élaboration du sens des textes). Mais l'usage de ces supports composites, dont la spécificité réside, nous le rappelons, dans une double hétérogénéité, simultanément sémiotique et discursive, dépasse largement le cadre des activités littéraires, puisque nous en retrouvons de semblables dans d'autres activités, relevant par exemple de l'histoire ou des sciences (voir notamment Viriot-Goeldel et Delarue-Breton [9], à paraître). Les travaux que nous menons sur les supports autres que littéraires aboutissent aux mêmes conclusions : il existe une corrélation nette entre le milieu social des élèves et la manière dont ils s'emparent de ces documents au cours des activités qui leur sont proposées au sein de la classe, dans la manière dont ils en construisent une signification et la mettent en mots. Ils ne s'agit donc bien évidemment pas de mettre en cause des modes de socialisation familiale, a fortiori de penser qu'ils suffisent à expliquer des inégalités scolaires, qui relèvent de processus fort complexes (voir l'ensemble de nos travaux qui pointent les phénomènes de coconstruction des inégalités au sein de la classe depuis plus de 20 ans), mais de mettre en évidence les lieux précis de difficultés potentielles socialement situées en lien avec les supports, afin de permettre aux enseignants de les identifier. Au delà de la littérature, la question des supports composites proposés au cours d'activités scolaires relevant de disciplines variées et de leur prise en compte par les enseignants nous semble ainsi mériter une attention particulière, au plan scientifique comme au plan pédagogique.

\section{Références}

[1] É. Bautier \& P. Rayou, Les inégalités d'apprentissages. Programmes, pratiques et malentendus scolaires. Paris : Presses Universitaires de France (2011)

[2] J.-Y. Rochex \& J. Crinon (Dir.), La construction des inégalités scolaires. Au cœur des pratiques et des dispositifs d'enseignement. Rennes : Presses Universitaires de Rennes (2011) 


\section{SHS Web of Conferences}

[3] É. Bautier É. \& J.-Y. Rochex, Activités conjointes ne signifie pas significations partagées. Raison éducative, 8, 199-220 (2004)

[4] É. Bautier É. \& R. Goigoux R., Difficultés d'apprentissage, processus de secondarisation et pratiques enseignantes : une hypothèse relationnelle. Revue Française de Pédagogie, 148, 89100 (2004)

[5] É. Bautier, J. Crinon, C. Delarue-Breton \& B. Marin, Des textes composites : des exigences de travail peu enseignées ? Repère, 45, 63-79 (2012)

[6] B. Bernstein, Class, codes and control, vol. 4. London : Routledge and Kegan Paul (1990)

[7] S. Bonnéry, Sociologie des dispositifs pédagogiques : structuration matérielle et technique, conceptions sociales de l'élève et apprentissages inégaux. In J.-Y. Rochex \& J. Crinon, La construction des inégalités scolaires. Au cœur des pratiques et des dispositifs d'enseignement. Rennes : Presses Universitaires de Rennes, 133-146 (2011)

[8] P. Richard-Principalli \& M.-F. Fradet, Complexité littéraire et littératie au début de l'école élémentaire, Symposium «Supports didactiques, pratiques d'enseignement et préoccupations sociologiques sur les inégalités d'apprentissage », XVIIe Congrès international de l'AMSE (Association mondiale des sciences de l'éducation), 3-8 juin, Reims (2012)

[9] C. Viriot-Goeldel C. \& C. Delarue-Breton C., Des textes composites à l'école : nouvelle littératie scolaire, apprentissages et inégalités. Spirale, 53 (2013, à paraître)

[10] É. Bautier, Pédagogie invisible et littératie étendue. Construire des significations scolaires à partir de supports composites. Communication au congrès de l'Association Française de Sociologie, Nantes, 2-5 septembre (2013)

[11] S. Scribner \& M. Cole, La littératie sans l'école : à la recherche des effets intellectuels de l'écriture. Langage et société, 133, 25-44 (2010)

[12] C. Delarue-Breton, Discours scolaire et paradoxe. Louvain : Academia-L'Harmattan (2012)

[13] C. Poslaniec, Vous avez dit « littérature »? Paris : Hachette (2002)

[14] U. Eco, Lector in fabula. Le rôle du lecteur ou la coopération interprétative dans les textes narratifs. Trad. M. Bouzaher. Paris : Grasset (1979) 